\title{
The impact of statin use on pneumonia risk and outcome: a combined population-based case- control and cohort study
}

Anders Gunnar Nielsen, Rikke Beck Nielsen, Anders Hammerich Riis, Søren Paaske Johnsen, Henrik Toft Sørensen and Reimar Wernich Thomsen*

\begin{abstract}
Introduction: The impact of statin use on pneumonia risk and outcome remains unclear. We therefore examined this risk in a population-based case-control study and did a 5-year update of our previous 30-day mortality analyses.

Methods: We identified 70,953 adults with a first-time hospitalization for pneumonia between 1997 and 2009 in Northern Denmark. Ten age- and sex-matched population controls were selected for each pneumonia patient. To control for potential confounders, we retrieved individual-level data on other medications, comorbidities, recent surgery, socioeconomic indicators, influenza vaccination, and other markers of frailty or health awareness from medical databases. We followed all pneumonia patients for 30 days after hospital admission.

Results: A total of 7,223 pneumonia cases (10.2\%) and 64523 controls (9.1\%) were statin users before admission, corresponding to an age- and sex-matched odds ratio (OR) of 1.17 (95\% confidence interval [Cl]: 1.14-1.21). After controlling for higher comorbidity and a wide range of other potential confounders, the adjusted OR for pneumonia associated with current statin use dropped to 0.80 (95\% Cl: 0.77-0.83). Previous statin use was not associated with decreased pneumonia risk (adjusted $\mathrm{OR}=0.97,95 \% \mathrm{Cl}: 0.91-1.02$ ). Decreased risk remained significant after further adjustment for frailty and health awareness markers.

The prevalence of statin use among Danish pneumonia patients increased from 1\% in 1997 to $24 \%$ in 2009. Thirtyday mortality following pneumonia hospitalization was $11.3 \%$ among statin users versus $15.1 \%$ among nonusers. This corresponded to a $27 \%$ reduced mortality rate (adjusted hazard ratio $=0.73,95 \% \mathrm{Cl}$ : $0.67-0.79$ ), corroborating our earlier findings.
\end{abstract}

Conclusions: Current statin use was associated with both a decreased risk of hospitalization for pneumonia and lower 30-day mortality following pneumonia.

\section{Introduction}

In addition to having a well-known cholesterol-lowering effect, statins regulate the immune and coagulation systems and prevent endothelial cell dysfunction [1-4]. These beneficial pleiotropic effects may explain why statin use has been associated with decreased risk and improved outcome of sepsis and other severe bacterial infections in a range of observational studies [5-9]. However, studies have reported conflicting results regarding the association

\footnotetext{
* Correspondence: rwt@dce.au.dk

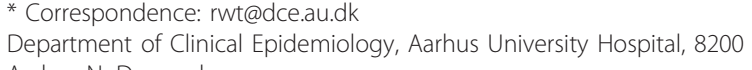
Aarhus N, Denmark
}

between statin use and pneumonia risk. Although four studies reported a reduced risk [10-13], one US study showed an increased risk and suggested that a 'healthyuser effect' in statin users may have accounted for the earlier results [14]. Regarding pneumonia prognosis, we previously reported a $31 \%$ lower mortality among statin users following pneumonia hospitalization in a population-based study [15]. These findings are in line with several other studies, including large population-based cohorts [16-22]. However, one Canadian study showed no reduction in mortality among statin users hospitalized for pneumonia [23], and a recent US study found that statin 
use did not protect against severe sepsis or mortality in hospitalized patients with pneumonia [24]. Evidence thus remains conflicting, leaving open the possibility of uncontrolled confounding by healthy-user effects in statin users [25]. Importantly, the prevalence of statin use in Denmark and elsewhere has increased dramatically in recent years, resulting in less selection of patients receiving statins.

Since large clinical trials that address this issue are still lacking [26], we conducted the hitherto largest population-based case-control study on statin use and risk of pneumonia, taking advantage of new high-quality Danish data to better control for confounders. We also updated previous 30-day pneumonia mortality analyses [15].

\section{Materials and methods Setting}

We conducted this study in Northern Denmark, which has 1.8 million inhabitants (30\% of Denmark's population). All citizens in Denmark are provided with universal tax-supported health care, which guarantees unfettered access to primary and secondary care that is free at the point of delivery and provides partial reimbursement for most prescribed medications, including statins. The unique Danish civil registration number permits cross-linking of individual-level data from different medical databases.

\section{Patients with pneumonia}

We identified patients with pneumonia from the Danish National Registry of Patients (DNRP), which contains data on inpatient discharges from all non-psychiatric hospitals in Denmark since 1977 and on emergency department and hospital outpatient clinic visits since 1995. Each contact is associated with one primary diagnosis and one or more secondary diagnoses classified according to the eighth revision of the International Classification of Diseases (ICD-8) until the end of 1993 and 10th revision (ICD-10) thereafter [27]. We included a total of 70,953 patients who were at least 15 years old with a first-time hospitalization for pneumonia during the study period (1 January 1997 to 12 December 2009). We excluded patients who had lived in the study region for less than 12 months.

\section{Population controls}

We used the Danish Civil Registration System (CRS) to select 10 population controls for each case; the controls were individually matched on age (birth year), gender, index date, and place of residence. The CRS has maintained vital statistics data, including date of birth, change of address, date of emigration, and exact date of death for the Danish population since 1968. We selected controls by using risk set sampling: each control had to be alive and at risk for a first pneumonia hospitalization on the day of admission (the index date) of the case to whom he or she was matched. In total, it was possible to match 70,914 pneumonia patients with 709,140 population controls for the risk analysis. In the updated cohort outcome analyses, all 70,954 patients with pneumonia were followed up for 30 days after hospital admission, with all-cause mortality as the outcome.

\section{Statin use}

Statins are available only by prescription in Denmark. As in our previous cohort study, current statin use was defined as at least one filled prescription within 125 days of the pneumonia hospitalization/index date [15]. Persons who filled a prescription more than 125 days beforehand were classified as former statin users. The 125-day period was chosen to capture most current users; if an $80 \%$ to $100 \%$ compliance rate is assumed, few statin prescriptions are expected to last beyond 125 days [28]. The prescription database contains information - including civil registration numbers, type and amount of drugs dispensed, and prescription dates - on reimbursable drugs dispensed at all pharmacies in Northern Denmark. In-hospital drug data were not available to allow an evaluation of the effectiveness of inpatient statin treatment in the cohort study.

\section{Covariates}

To control for comorbidities that may be associated with pneumonia, we obtained data from the DNRP on all previous hospital diagnoses for each individual since 1977. We obtained data on the 19 disease categories that comprise the Charlson comorbidity index [29-31]. The DNRP also provided information on hospital-diagnosed obesity, alcoholism-related disorders within 1 year of the index date, and surgical procedures performed within 3 months of the index date. In addition, we obtained data on the use of the following preadmission medications, which may reflect important underlying comorbidities and/or affect either risk or prognosis of pneumonia: immunosuppressive drugs and oral steroids within 1 year of admission, systemic antibiotic therapy within 3 months of admission (for outcome analyses only), concurrent use (within 125 days of the index date) of inhaled corticosteroid therapy, inhaled beta 2 agonists, combination of inhaled steroids and beta 2 agonists, beta blockers, lowdose aspirin, loop diuretics, thiazides, angiotensinconverting enzyme inhibitors, angiotensin receptor antagonists, digoxin, vitamin $\mathrm{K}$ antagonists, calcium channel blockers, nitrates, antipsychotics, non-steroidal anti-inflammatory drugs, and proton-pump inhibitors. For ICD and Anatomical Therapeutic Chemical prescription codes, see Additional files 1 and 2.

To control for socioeconomic confounding, we obtained data from the CRS on marital status (married, divorced or widowed, never married, or unknown) and 
urbanization (city with more than 100,000 inhabitants, provincial town with 10,000 to 99,999 inhabitants, or rural area with fewer than 10,000 inhabitants).

To further control for a possible healthy-user effect in statin users, we obtained information from the National Health Insurance Service Registry for the period of 1 January 2001 to 12 December 2009 (for details, see Additional file 3). This registry contains detailed information on all patient contacts with general practitioners (GPs) and specialist practitioners in the primary-care sector. We retrieved data on receipt of the current season's reimbursed influenza vaccine prior to the pneumonia index date and on the following markers of health awareness or frailty: GP preventive consultations and services, social medicine-related GP consultations, GP conversational therapy, application for reimbursement for chronic and terminal illness, and GP diagnosis and treatment of dementia.

\section{Statistical analysis}

In our setting with a relatively low proportion of treated subjects and outcome events and many time-varying covariates, we chose an incidence case-control study design with risk set sampling instead of a cohort design to study pneumonia risk $[32,33]$. In the case-control study, we used conditional logistic regression to estimate the odds ratio (OR) for hospitalization with pneumonia among current and former users of statins in comparison with never users. The controls were matched on age, sex, place of residence, and index date. We adjusted for the following covariates: individual comorbidities, preadmission drugs (except for systemic antibiotic therapy), socioeconomic indicators, living with small children, and (for the subcohort of cases and controls for 2001-2009) influenza vaccination and other markers of health awareness or frailty. Because we used risk set sampling of controls, the ORs provided unbiased estimates of incidence rate ratios.

In the cohort study, we used Cox regression analysis to compute 30-day mortality rate ratios (MRRs), controlling for sex, age, place of residence as well as the same covariates used in the case-control analysis, except for exclusion of living with small children and inclusion of preadmission antibiotic therapy in the prognosis analysis model.

We repeated both risk and outcome analyses while stratifying on calendar time periods, comorbidity levels, age groups, and medical indication for statin use in the main model (1997-2009). The risk estimates associated with simvastatin, atorvastatin, pravastatin, and other statins were assessed separately. In the subcohort analysis for 2001-2009, we further restricted the analyses to patients who received the current year's influenza vaccine and had different markers of frailty and health awareness. In the stratified analyses, the matching was retained.
Finally, we examined a potential dose-response association by calculating the number of defined daily doses (DDDs) of statin received within 125 days of the index date for each patient. The adjusted ORs and MRRs were plotted against statin DDD percentiles. Data were obtained from Danish health-care registries, which are generally available to researchers; according to Danish law, use of these registries does not require informed consent. The study was approved by the Danish Data Protection Agency (record number 2009-41-3866).

\section{Results}

\section{Case-control study of pneumonia risk}

We identified 70,914 cases of patients hospitalized for pneumonia and 709,140 population controls. Current statin users comprised 7,223 (10.2\%) of the cases and $64,523(9.1 \%)$ of the controls. Among current statin users, $78 \%$ received simvastatin, $12 \%$ received atorvastatin, $5.5 \%$ received pravastatin, and $4.5 \%$ received other statins. Slightly more than half of the cases were male; their median age was 73 years, and two thirds were older than 65 years (Table 1, case-control study). Compared with controls, pneumonia cases were more likely to have comorbidities, particularly cardiovascular disease, chronic pulmonary disease, cancer, or recent surgery. Medication use was also much more common among cases than controls, particularly diuretics and other cardiovascular therapies and corticosteroids. However, influenza vaccination was only slightly more common among cases.

The unadjusted age- and sex-matched OR associating current statin use with pneumonia occurrence was 1.17 (95\% confidence interval (CI) 1.14 to 1.21) (Table 2, casecontrol study). Adjustment for potential confounders reduced the OR to 0.80 ( $95 \%$ CI 0.77 to 0.83 ). No association was found between former statin use and the risk of pneumonia (adjusted OR $=0.97,95 \%$ CI 0.91 to 1.02 ). The adjusted ORs associated with use of simvastatin, atorvastatin, and pravastatin were 0.80 ( $95 \% \mathrm{CI} 0.77$ to 0.83 ), 0.83 ( $95 \%$ CI 0.76 to 0.91 ), and 0.86 (95\% CI 0.76 to 0.98 ), respectively. For the period of 2001-2009, we further adjusted for receipt of influenza vaccine and markers of frailty and health awareness. This step left the OR of pneumonia associated with current statin use virtually unchanged (adjusted OR $=0.77,95 \%$ CI 0.74 to 0.80 ) (Table 2, case-control study), whereas the adjusted OR for former statin use was 0.93 (95\% CI 0.87 to 0.98 ).

We adjusted for important confounders separately to evaluate their importance in current statin users (data not shown). Comorbidity (individual adjustment for the 19 disease categories in the Charlson index) appeared to be the most important confounder (comorbidity-only adjusted $\mathrm{OR}=0.86,95 \% \mathrm{CI} 0.84$ to 0.89 ). Adjusting only for the use of 18 different medications yielded an OR of 0.90 (95\% CI 0.87 to 0.93). Adjusting only for markers of 
Table 1 Case-control study: characteristics of 70,914 hospitalized patients with pneumonia and 709,140 population controls in Denmark (1997-2009)

\begin{tabular}{|c|c|c|c|}
\hline Characteristics at time of hospital admission & $\begin{array}{l}\text { Pneumonia cases } \\
(n=70,914)\end{array}$ & $\begin{array}{l}\text { Population controls } \\
(\mathrm{n}=709,140)\end{array}$ & $\begin{array}{c}\text { Total } \\
(n=780,045)\end{array}$ \\
\hline \multicolumn{4}{|l|}{ Age, number (percentage) } \\
\hline $15-39$ years & $5,140(7.3)$ & $51,601(7.3)$ & $56,741(7.3)$ \\
\hline 40-64 years & $17,803(25.1)$ & $178,095(25.1)$ & $195,898(25.1)$ \\
\hline $65-79$ years & $26,122(36.8)$ & $261,046(36.8)$ & $287,168(36.8)$ \\
\hline$\geq 80$ years & $21,849(30.8)$ & $218,398(30.8)$ & $240,247(30.8)$ \\
\hline \multicolumn{4}{|l|}{ Sex, number (percentage) } \\
\hline Female & $33,278(46.9)$ & $332,780(46.9)$ & $366,058(46.9)$ \\
\hline Male & $37,636(53.1)$ & $376,360(53.1)$ & $413,996(53.1)$ \\
\hline \multicolumn{4}{|l|}{ Calendar period, number (percentage) } \\
\hline $1997-2000$ & $17,754(25.0)$ & $177,540(25.0)$ & $195,294(25.0)$ \\
\hline $2001-2004$ & $23,786(33.5)$ & $237,860(33.5)$ & $261,646(33.5)$ \\
\hline 2005-2009 & $29,374(41.4)$ & $293,740(41.4)$ & $323,144(41.4)$ \\
\hline \multicolumn{4}{|l|}{ Preadmission hospital-diagnosed comorbidities, number (percentage) } \\
\hline \multicolumn{4}{|l|}{ Conditions included in the Charlson index } \\
\hline Previous myocardial infarction & $6,704(9.5)$ & $39,573(5.6)$ & $46,277(5.9)$ \\
\hline Congestive heart failure & $7,457(10.5)$ & $29,791(4.2)$ & $37,248(4.8)$ \\
\hline Peripheral vascular disease & $6,100(8.6)$ & $28,615(4.0)$ & $34,715(4.5)$ \\
\hline Cerebrovascular disease & $10,672(15.0)$ & $62,515(8.8)$ & $73,187(9.4)$ \\
\hline Dementia & $1,581(2.2)$ & $9,343(1.3)$ & $10,924(1.4)$ \\
\hline Hemiplegia & $472(0.7)$ & $1,133(0.2)$ & $1,605(0.2)$ \\
\hline Chronic pulmonary disease & $14,457(20.4)$ & $44,225(6.2)$ & $58,682(7.5)$ \\
\hline Connective tissue disease & $3,759(5.3)$ & $19,158(2.7)$ & $22,917(2.9)$ \\
\hline Peptic ulcer disease & $6,198(8.7)$ & $34,861(4.9)$ & $41,059(5.3)$ \\
\hline Mild liver disease & 1,299 (1.8) & $3,671(0.5)$ & $4,970(0.6)$ \\
\hline Moderate to severe liver disease & $386(0.54)$ & $861(0.12)$ & $1,247(0.2)$ \\
\hline Diabetes without end-stage organ damage & $5,662(8.0)$ & $28,185(4.0)$ & $33,847(4.3)$ \\
\hline Diabetes with end-stage organ damage & $2,978(4.2)$ & $11,478(1.6)$ & $14,456(1.9)$ \\
\hline Moderate to severe renal disease & $2,714(3.8)$ & $8,523(1.2)$ & $11,237(1.4)$ \\
\hline Solid cancer & $10,737(15.1)$ & $62,092(8.8)$ & $72,829(9.3)$ \\
\hline Metastatic solid cancer & $2,043(2.9)$ & $4,174(0.6)$ & $6,217(0.8)$ \\
\hline Leukemia & $744(1.0)$ & $1,267(0.2)$ & $2,011(0.3)$ \\
\hline Lymphoma & $1,307(1.8)$ & $2,490(0.4)$ & $3,797(0.5)$ \\
\hline AIDS & $88(0.1)$ & $87(0.0)$ & $175(0.0)$ \\
\hline \multicolumn{4}{|l|}{ Charlson comorbidity index score } \\
\hline Low, 0 & $26,285(37.1)$ & $457,185(64.5)$ & $483,470(62.0)$ \\
\hline Medium, 1-2 & $28,627(40.4)$ & $197,723(27.9)$ & $226,350(29.0)$ \\
\hline High, $\geq 3$ & $16,002(22.6)$ & $54,232(7.7)$ & $70,234(9.0)$ \\
\hline \multicolumn{4}{|l|}{ Additional comorbidity } \\
\hline Alcoholism-related disorders & $4,303(6.1)$ & $13,871(2.0)$ & $18,174(2.3)$ \\
\hline Obesity & $2,869(4.1)$ & $12,515(1.8)$ & $15,384(2.0)$ \\
\hline Any surgical procedure within 90 days of index date & $14,782(20.8)$ & $40,385(5.7)$ & $55,167(7.1)$ \\
\hline \multicolumn{4}{|c|}{ Preadmission medications within 125 days of index date, unless otherwise indicated } \\
\hline Statins, current use & $7,223(10.2)$ & $64,523(9.1)$ & $71,746(9.2)$ \\
\hline Statins, former use (>125 days) & $1,908(2.7)$ & $14,102(2.0)$ & $16,010(2.1)$ \\
\hline Oral steroids (within 1 year of index date) & $13,124(18.5)$ & $45,763(6.5)$ & $58,887(7.6)$ \\
\hline Other immunosuppressive drugs (within 1 year of index date) & $1,347(1.9)$ & $4,789(0.7)$ & $6,136(0.8)$ \\
\hline \multicolumn{4}{|l|}{ Users of inhalation medications } \\
\hline Beta2 agonists only & $5,514(7.8)$ & $15,110(2.1)$ & $20,624(2.6)$ \\
\hline Corticosteroid therapy only & $823(1.2)$ & $5,463(0.8)$ & $6,286(0.8)$ \\
\hline Corticosteroid therapy and beta2 agonists & $9,119(12.9)$ & $25,835(3.6)$ & $34,954(4.5)$ \\
\hline Beta blockers & $11,988(16.9)$ & $93,125(13.1)$ & $105,113(13.5)$ \\
\hline Low-dose aspirin & $12,731(18.0)$ & $95,825(13.5)$ & $108,556(13.9)$ \\
\hline
\end{tabular}


Table 1 Case-control study: characteristics of 70,914 hospitalized patients with pneumonia and 709,140 population controls in Denmark (1997-2009) (Continued)

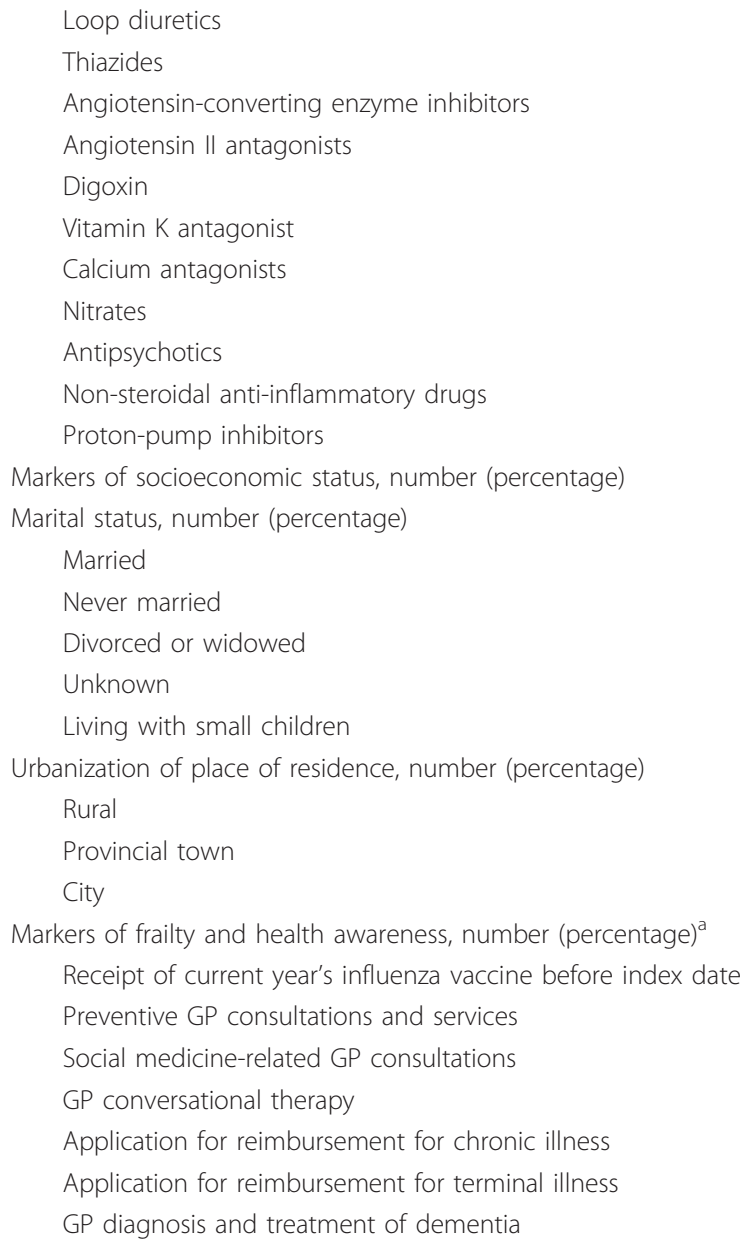

$\begin{array}{ccc}17,804(25.1) & 76,134(10.7) & 93,938(12.0) \\ 9,432(13.3) & 85,475(12.0) & 94,907(12.2) \\ 9,309(13.1) & 73,417(10.4) & 82,726(10.6) \\ 4,598(6.5) & 46,242(6.5) & 50,840(6.5) \\ 6,071(8.6) & 32,810(4.6) & 38,881(5.0) \\ 4,024(5.7) & 22,590(3.2) & 26,614(3.4) \\ 10,466(14.8) & 84,077(11.9) & 94,543(12.1) \\ 6,384(9.0) & 34,384(4.9) & 40,768(5.2) \\ 4,902(6.9) & 21,013(3.0) & 25,915(3.3) \\ 12,641(17.8) & 87,279(12.3) & 99,920(12.8) \\ 11,469(16.2) & 50,146(7.1) & 61,615(7.9)\end{array}$

$\begin{array}{ccc}33,423(47.1) & 376,521(53.1) & 409,944(52.6) \\ 8,957(12.6) & 80,230(11.3) & 89,187(11.4) \\ 28,348(40.0) & 250,837(35.4) & 279,185(35.8) \\ 186(0.3) & 1,552(0.2) & 1,738(0.2) \\ 940(1.3) & 7,981(1.1) & 8,921(1.1) \\ & & \\ 14,138(20.0) & 143,517(20.2) & 157,655(20.2) \\ 37,909(53.5) & 384,034(54.2) & 421,943(54.1) \\ 18,867(26.6) & 181,589(25.6) & 200,456(25.7) \\ & & \\ 9,538(17.9) & 74,873(14.1) & 84,411(14.4) \\ 5,863(11.0) & 37,349(7.0) & 43,212(7.0) \\ 15,118(28.4) & 59,686(11.2) & 74,804(12.8) \\ 5,124(9.6) & 18,738(3,5) & 23,862(4.1) \\ 2,703(5.1) & 5,958(1.1) & 8,661(1.5) \\ 274(0.5) & 229(0.04) & 503(0.1) \\ 560(1.1) & 4,046(0.8) & 4,606(0.8)\end{array}$

${ }^{a}$ Ascertained among 53,160 pneumonia cases who could be matched with 531,600 controls for 2001-2009. GP, general practitioner.

frailty and health awareness yielded an OR of 1.03 (95\% CI 1.00 to 1.06 ).

\section{Cohort study of 30-day mortality after hospitalization for pneumonia}

The prevalence of current statin use among patients with pneumonia increased dramatically from 1997 (1\%) to 2009 (24\%). Thus, $74 \%$ of all statin users in our
1997-2009 cohort were hospitalized after 2004, which was the last year of study in our previous mortality analysis [15].

Characteristics of statin users and non-users with pneumonia in the cohort study are presented in Table 3. Statin users were less likely to be at the extremes of age but had much more previous cardiovascular disease and diabetes. Of importance, they also had higher rates of

Table 2 Case-control study: preadmission statin use and risk of hospitalization for pneumonia in Denmark (1997-2009)

\begin{tabular}{|c|c|c|c|c|}
\hline Statin use & $\begin{array}{l}\text { Pneumonia cases } \\
(n=70,914)\end{array}$ & $\begin{array}{l}\text { Population controls } \\
(\mathrm{n}=709,140)\end{array}$ & $\begin{array}{l}\text { Crude }^{\mathrm{a}} \text { OR } \\
(95 \% \mathrm{Cl})\end{array}$ & $\begin{array}{l}\text { Adjusted }^{\text {b }} \text { OR } \\
(95 \% \mathrm{Cl})\end{array}$ \\
\hline Never & $61,783(87.1 \%)$ & $630,515(88.9 \%)$ & 1.00 (reference) & 1.00 (reference) \\
\hline Former ( $>125$ days before admission) & $1,908(2.7 \%)$ & $14,102(2.0 \%)$ & $1.42(1.35-1.50)$ & $0.97(0.91-1.02)$ \\
\hline Current ( $\leq 125$ days before admission) & $7,223(10.2 \%)$ & $64,523(9.1 \%)$ & $1.17(1.14-1.21)$ & $0.80(0.77-0.83)$ \\
\hline $\begin{array}{l}\text { Current ( } \leq 125 \text { days before admission), } \\
\text { supplementary adjusted model for 2001-2009 }\end{array}$ & $6,883(13.0 \%)$ & $61,450(11.6 \%)$ & $1.18(1.15-1.21)$ & $0.77(0.74-0.80)$ \\
\hline
\end{tabular}

${ }^{a}$ Matched for age, sex, urbanization of place of residence, and hospitalization/index date. ${ }^{b}$ Adjusted for marital status, calendar period, living with small children, any recent surgical procedure, 20 different comorbidities, alcoholism-related conditions, and 18 different preadmission medications (Table 1). The supplementary model for 2001-2009 includes 53,160 pneumonia cases and 531,600 controls and is also adjusted for receipt of current year's influenza vaccine, dementia, social medicine-related consultations, application for reimbursement for chronic or terminal illness, conversational therapy, and general practitioner preventive consultations and services. $\mathrm{Cl}$, confidence interval; $\mathrm{OR}$, odds ratio. 
Table 3 Cohort study: characteristics of 70,953 patients hospitalized for pneumonia for the first time in Denmark according to statin use (1997-2009)

\begin{tabular}{|c|c|c|c|c|}
\hline $\begin{array}{l}\text { Characteristics at time of pneumonia } \\
\text { admission }\end{array}$ & $\begin{array}{c}\text { Current } \\
\text { statin use } \\
\text { ( } \leq 125 \text { days) } \\
n=7,223 \\
(10.2 \%)\end{array}$ & $\begin{array}{c}\text { Former statin use } \\
\begin{array}{c}(>125 \text { days }) \\
n=1,903 \\
(2.7 \%)\end{array}\end{array}$ & $\begin{array}{c}\text { Never } \\
\text { statin use } \\
\mathrm{n}=61,827 \\
(87.1 \%)\end{array}$ & $\begin{array}{c}\text { Total } \\
\mathrm{n}=70,953 \\
(100 \%)\end{array}$ \\
\hline \multicolumn{5}{|l|}{ Age, number (percentage) } \\
\hline 15-39 years & $43(0.6)$ & $19(1.0)$ & $5078(8.2)$ & $5,140(7.2)$ \\
\hline $40-64$ years & $1,756(24.3)$ & $518(27.2)$ & $15,529(25.1)$ & $17,803(25.1)$ \\
\hline $65-79$ years & $3,819(52.9)$ & $929(48.8)$ & $21,374(34.6)$ & $26,122(36.8)$ \\
\hline$\geq 80$ years & $1,605(22.2)$ & $437(23.0)$ & $19,846(32.1)$ & $21,888(30.9)$ \\
\hline \multicolumn{5}{|l|}{ Sex, number (percentage) } \\
\hline Female & $3,047(42.2)$ & $855(44.9)$ & $29,389(47.5)$ & $33,291(46.9)$ \\
\hline Male & $4,176(57.8)$ & $1,048(55.1)$ & $32,438(52.5)$ & $37,662(53.1)$ \\
\hline \multicolumn{5}{|l|}{ Calendar period, number (percentage) } \\
\hline $1997-2000$ & $340(4.7)$ & $62(3.3)$ & $17,359(28.0)$ & $17,761(25.0)$ \\
\hline $2001-2004$ & $1,520(21.0)$ & $318(16.7)$ & $21,957(36.0)$ & $23,795(33.5)$ \\
\hline $2005-2009$ & $5,363(74.3)$ & $1,523(80.0)$ & $22,511(36.4)$ & $29,397(41.4)$ \\
\hline \multicolumn{5}{|l|}{$\begin{array}{l}\text { Preadmission hospital-diagnosed comorbidities, } \\
\text { number (percentage) }\end{array}$} \\
\hline \multicolumn{5}{|l|}{ Conditions included in Charlson index } \\
\hline Previous myocardial infarction & $2,346(32.5)$ & $482(25.3)$ & $3,877(6.2)$ & $6,705(9.5)$ \\
\hline Congestive heart failure & $1,443(20.0)$ & $358(18.8)$ & $5,661(9.2)$ & $7,462(10.5)$ \\
\hline Peripheral vascular disease & $1,506(20.9)$ & $389(20.4)$ & $4,206(6.8)$ & $6,101(8.6)$ \\
\hline Cerebrovascular disease & $2,030(28.1)$ & $495(26.0)$ & $8,151(13.2)$ & $10,676(15)$ \\
\hline Dementia & $134(1.9)$ & $36(1.9)$ & $1,412(2.3)$ & $1,582(2.23)$ \\
\hline Hemiplegia & $36(0.5)$ & $6(0.3)$ & $430(0.7)$ & $472(0.7)$ \\
\hline Chronic pulmonary disease & $1,728(23.9)$ & $431(22.7)$ & $12,299(19.9)$ & $14,458(20.4)$ \\
\hline Connective tissue disease & $441(6.1)$ & $134(7.0)$ & $3,185(5.2)$ & $3,760(5.3)$ \\
\hline Peptic ulcer disease & $735(10.2)$ & $215(11.3)$ & $5,253(8.5)$ & $6,203(8.4)$ \\
\hline Mild liver disease & $82(1.1)$ & $52(2.7)$ & $1,165(1.9)$ & $1,299(1.8)$ \\
\hline Moderate to severe liver disease & $17(0.2)$ & $22(1.2)$ & $347(0.6)$ & $386(0.5)$ \\
\hline Diabetes without end-stage organ damage & $1,505(20.8)$ & $392(20.6)$ & $3,766(6.0)$ & $5,663(8.0)$ \\
\hline Diabetes with end-stage organ damage & $942(13.0)$ & $242(12.7)$ & $1,794(2.9)$ & $2,978(4.2)$ \\
\hline Moderate to severe renal disease & $532(7.4)$ & $189(9.9)$ & $1,993(3.2)$ & 2,714 (3.8) \\
\hline Solid cancer & $1,114(15.4)$ & $384(20.2)$ & $9,242(15)$ & $10,740(15.1)$ \\
\hline Metastatic solid cancer & $195(2.7)$ & $87(4.6)$ & $1,761(2.9)$ & $2,043(2.9)$ \\
\hline Leukemia & $53(0.7)$ & $19(1.0)$ & $672(1.1)$ & $744(1.1)$ \\
\hline Lymphoma & $98(1.4)$ & $53(2.8)$ & $1,157(1.9)$ & $1,308(1.8)$ \\
\hline AIDS & $3(0.0)$ & $0(0.0)$ & $85(0.1)$ & $88(0.1)$ \\
\hline \multicolumn{5}{|l|}{ Charlson comorbidity index score } \\
\hline Low, 0 & $1,052(14.6)$ & $290(15.2)$ & $24,966(40.4)$ & $26,308(37.0)$ \\
\hline Medium, 1-2 & $3,259(45.0)$ & $772(40.6)$ & $24,609(39.8)$ & $28,640(40.4)$ \\
\hline High, $\geq 3$ & $2,912(40.3)$ & $841(44.2)$ & $12,252(19.8)$ & $16,005(22.6)$ \\
\hline \multicolumn{5}{|l|}{ Additional comorbidity } \\
\hline Alcoholism-related disorders & $352(4.9)$ & $135(7.1)$ & $3,813(6.2)$ & $4,300(6.1)$ \\
\hline Obesity & $588(8.1)$ & $137(7.2)$ & $1,906(3.1)$ & $2,631(3.7)$ \\
\hline $\begin{array}{l}\text { Any surgical procedure within } 90 \text { days of index } \\
\text { date }\end{array}$ & $1,970(27.3)$ & $518(27.2)$ & $12,298(19.9)$ & $14,786(20.8)$ \\
\hline \multicolumn{5}{|l|}{$\begin{array}{l}\text { Preadmission medications within } 125 \text { days of index } \\
\text { date unless otherwise indicated, number (percentage) }\end{array}$} \\
\hline $\begin{array}{l}\text { Immunosuppressive drugs (within } 1 \text { year of index } \\
\text { date) }\end{array}$ & $157(2.2)$ & $49(2.6)$ & $1,141(1.9)$ & $1,347(1.9)$ \\
\hline Oral steroids (within 1 year of index date) & $1,459(20.2)$ & $436(22.9)$ & $11,230(18.2)$ & $13,125(18.5)$ \\
\hline $\begin{array}{l}\text { Systemic antibiotic therapy (within } 90 \text { days of } \\
\text { index date) }\end{array}$ & $2,995(41.5)$ & $790(41.5)$ & $24,978(40.4)$ & $28,763(40.5)$ \\
\hline
\end{tabular}


Table 3 Cohort study: characteristics of 70,953 patients hospitalized for pneumonia for the first time in Denmark according to statin use (1997-2009) (Continued)

\begin{tabular}{|c|c|c|c|c|}
\hline \multicolumn{5}{|l|}{ Users of inhalation medications } \\
\hline Beta2 agonists only & $509(7.0)$ & $153(8.0)$ & $4,854(7.9)$ & $5,516(7.8)$ \\
\hline Corticosteroid therapy only & $86(1.2)$ & $18(1.0)$ & $719(1.2)$ & $823(1.2)$ \\
\hline Corticosteroids and beta2 agonists & $1,105(15.3)$ & $257(13.5)$ & $7,757(12.6)$ & $9,119(12.9)$ \\
\hline Beta blockers & $3,290(45.6)$ & $579(30.4)$ & $8,124(13.1)$ & $11,993(16.9)$ \\
\hline Low-dose aspirin & $3,473(48.1)$ & $665(35.0)$ & $8,605(13.9)$ & $12,743(18.0)$ \\
\hline Loop diuretics & $2,672(37.0)$ & $589(30.1)$ & $14,558(23.6)$ & $17,819(25.1)$ \\
\hline Thiazides & $1,347(18.7)$ & $309(16.2)$ & $7,782(12.6)$ & $9,438(13.3)$ \\
\hline Angiotensin-converting enzyme inhibitor & $2,512(34.8)$ & $462(24.3)$ & $6,339(10.3)$ & $9,313(13.1)$ \\
\hline Angiotensin II antagonists & $1,273(17.6)$ & $289(15.2)$ & $3,037(4.9)$ & $4,599(6.5)$ \\
\hline Digoxin & $671(9.3)$ & $151(7.9)$ & $5,252(8.5)$ & $6,074(8.6)$ \\
\hline Vitamin $\mathrm{K}$ antagonist & $927(12.8)$ & $179(9.4)$ & $2,918(4.7)$ & $4,024(5.7)$ \\
\hline Calcium antagonists & $2,261(31.3)$ & $467(24.5)$ & $7,742(12.5)$ & $10,470(14.8)$ \\
\hline Nitrates & $1,658(23.0)$ & $293(15.4)$ & $4,436(7.2)$ & $6,387(9.0)$ \\
\hline Antipsychotics & $397(5.5)$ & $115(6.0)$ & $4,392(7.1)$ & $4,904(6.9)$ \\
\hline Non-steroidal anti-inflammatory drugs & $1,341(18.6)$ & $349(18.3)$ & $10,953(17.7)$ & $12,643(17.8)$ \\
\hline Proton-pump inhibitors & $1,860(25.8)$ & $542(28.5)$ & $9,071(14.7)$ & $11,473(16.2)$ \\
\hline \multicolumn{5}{|l|}{ Markers of socioeconomic status, number (percentage) } \\
\hline \multicolumn{5}{|l|}{ Marital status, number (percentage) } \\
\hline Married & $4,131(57.2)$ & $1,034(54.3)$ & $28,261(45.7)$ & $33,426(47.1)$ \\
\hline Never married & $463(6.4)$ & $102(5.4)$ & $8,393(13.6)$ & $8,958(12.6)$ \\
\hline Divorced or widowed & $2,614(36.2)$ & $764(40.2)$ & $25,005(40.4)$ & $28,383(40.0)$ \\
\hline Unknown & $15(0.2)$ & $3(0.2)$ & $168(0.3)$ & $186(0.3)$ \\
\hline \multicolumn{5}{|l|}{$\begin{array}{l}\text { Urbanization of place of residence, number } \\
\text { (percentage) }\end{array}$} \\
\hline Rural & $1,235(17.1)$ & $283(14.9)$ & $11,921(18.3)$ & $12,809(18.0)$ \\
\hline Provincial town & $4,054(56.1)$ & $1,055(55.4)$ & $34,083(55.1)$ & $39,192(55.2)$ \\
\hline City & $1,934(26.8)$ & $565(29.7)$ & $16,453(26.6)$ & $18,952(26.7)$ \\
\hline \multicolumn{5}{|l|}{$\begin{array}{l}\text { Markers of frailty or health awareness, number } \\
\text { (percentage) }^{\mathrm{a}}\end{array}$} \\
\hline $\begin{array}{l}\text { Receipt of current year's reimbursed influenza } \\
\text { vaccine before index date }\end{array}$ & $2,267(32.9)$ & $519(28.2)$ & $6,760(15.2)$ & $9,546(18.0)$ \\
\hline Preventive GP consultations and services & $1,867(27.1)$ & $528(28.7)$ & $3,472(7.8)$ & $5,867(11.0)$ \\
\hline Social medicine-related GP consultations & $1,967(28.6)$ & $578(31.4)$ & $12,589(28.3)$ & $15,134(28.5)$ \\
\hline GP conversational therapy & $780(11.3)$ & $237(12.9)$ & $4,108(9.2)$ & $5,125(9.6)$ \\
\hline Application for reimbursement for chronic illness & $670(9.7)$ & $145(7.9)$ & $1,888(4.3)$ & $2,703(5.1)$ \\
\hline Application for reimbursement for terminal illness & $28(0.4)$ & $21(1.1)$ & $225(0.5)$ & $274(0.5)$ \\
\hline GP diagnosis and treatment of dementia & $85(1.2)$ & $29(1.6)$ & $446(1.0)$ & $560(1.1)$ \\
\hline
\end{tabular}

${ }^{a}$ Ascertained among 53,192 pneumonia patients hospitalized in 2001-2009. GP, general practitioner.

chronic pulmonary disease and corticosteroid use, a similar prevalence rate of cancer, but less liver disease and alcoholism in comparison with non-users. Statin users were more likely to be married, receive influenza vaccinations, and have preventive consultations with GPs. Reimbursement rates for chronic or terminal illness or dementia were similar in statin users and non-users (Table 3, cohort study).

Our updated outcome analyses showed that cumulative 30-day mortality rates following pneumonia hospitalization were $11.3 \%$ among statin users and 15.1\% among non-users (Table 4, cohort study). Results of Cox regression analysis indicated a $27 \%$ decrease in pneumonia mortality associated with current statin use (adjusted hazard ratio (HR) $=0.73,95 \%$ CI 0.67 to 0.79 ); this finding is similar to our earlier findings [15]. Supplementary analyses that included influenza vaccination and other markers of frailty and health awareness for the 2001-2009 period did not materially alter this estimate (adjusted HR $=0.76,95 \%$ CI 0.70 to 0.83 ). In neither of these analyses was former statin use associated with mortality. 
Table 4 Cohort study: preadmission statin use and prognosis within $\mathbf{3 0}$ days following hospitalization for pneumonia in Denmark (1997-2009)

\begin{tabular}{|c|c|c|c|c|c|}
\hline Statin use & $\begin{array}{c}\text { Pneumonia } \\
\text { patients } \\
(n=70,953)\end{array}$ & $\begin{array}{l}\text { Number of } \\
\text { deaths }\end{array}$ & $\begin{array}{l}\text { 30-day mortality, } \\
\text { percentage }\end{array}$ & $\begin{array}{l}\text { Crude HR } \\
(95 \% \mathrm{Cl})\end{array}$ & $\begin{array}{l}\text { Adjusted }^{\mathrm{a}} \mathrm{HR} \\
(95 \% \mathrm{Cl})\end{array}$ \\
\hline Never & 61,827 & 9,282 & 15.1 & $\begin{array}{c}1.00 \\
\text { (reference) }\end{array}$ & $\begin{array}{c}1.00 \\
\text { (reference) }\end{array}$ \\
\hline Former (>125 days) & 1,903 & 272 & 14.3 & $0.95(0.84-1.07)$ & $0.91(0.80-1.03)$ \\
\hline Current ( $\leq 125$ days) & 7,223 & 813 & 11.3 & $0.74(0.69-0.79)$ & $0.73(0.67-0.79)$ \\
\hline $\begin{array}{l}\text { Current ( } \leq 125 \text { days) supplementary } \\
\text { adjusted model for 2001-2009 }\end{array}$ & 6,883 & 788 & 11.4 & $0.77(0.71-0.83)$ & $0.76(0.70-0.83)$ \\
\hline
\end{tabular}

adjusted model for 2001-2009

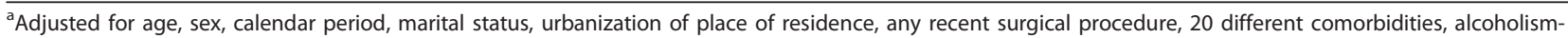
related conditions, and 19 different preadmission medications. The supplementary model for 2001-2009 is also adjusted for receipt of current year's influenza vaccine, dementia, social medicine-related consultations, application for reimbursement for chronic and terminal illness, conversational therapy, and preventive consultations and services. $\mathrm{Cl}$, confidence interval; $\mathrm{HR}$, hazard ratio.

\section{Additional analyses}

The results of additional subgroup analyses are presented in Table 5 (case-control and cohort study). In general, the risk and mortality reduction associated with current statin use was observed in most subgroups. However, in some strata, former statin use was also associated with some beneficial effect compared with never use, suggesting either residual confounding by healthy-user effects or a protracted long-term 'legacy' effect of statins. Finally, we found no clear doseresponse relationship between statin dose and pneumonia risk (Figure 1a) and 30-day mortality (Figure 1b), respectively. As Figure $1 \mathrm{~b}$ shows, consumption of approximately 1 statin DDD was associated with the lowest mortality.

\section{Discussion}

In this hitherto largest case-control study on statin use and risk of pneumonia, current statin use was associated with a reduced risk of hospitalization for communityacquired pneumonia. Moreover, we confirmed our previous finding of lower 30-day mortality following hospitalization with pneumonia among current statin users. Not only did pneumonia prognosis improve with statin

Table 5 Statin use and pneumonia risk and mortality within various patient subgroups

\begin{tabular}{|c|c|c|c|c|}
\hline \multirow{2}{*}{$\begin{array}{l}\text { Variables } \\
\text { Main model for 1997-2009 }\end{array}$} & \multicolumn{2}{|c|}{$\begin{array}{l}\text { Risk of hospitalization for } \\
\text { pneumonia, OR }(95 \% \mathrm{Cl})\end{array}$} & \multicolumn{2}{|c|}{$\begin{array}{l}\text { 30-day mortality after hospitalization } \\
\text { for pneumonia, HR }(95 \% \mathrm{Cl})\end{array}$} \\
\hline & Current statin use & Former statin use & Current statin use & Former statin use \\
\hline \multicolumn{5}{|l|}{ Calendar time } \\
\hline $1997-2000$ & $0.77(0.68-0.88)$ & $1.12(0.84-1.49)$ & $0.57(0.38-0.85)$ & $1.04(0.52-2.10)$ \\
\hline 2001-2004 & $0.79(0.74-0.85)$ & $0.88(0.77-1.00)$ & $0.70(0.59-0.83)$ & $0.95(0.70-1.29)$ \\
\hline 2005-2009 & $0.81(0.77-0.84)$ & $0.98(0.92-1.05)$ & $0.74(0.67-0.82)$ & $0.90(0.78-1.03)$ \\
\hline \multicolumn{5}{|l|}{ Charlson comorbidity index score } \\
\hline Low, 0 & $0.78(0.72-0.84)$ & $1.03(0.90-1.18)$ & $0.73(0.56-0.94)$ & $0.77(0.48-1.22)$ \\
\hline Medium, 1-2 & $0.74(0.70-0.78)$ & $0.83(0.76-0.91)$ & $0.73(0.64-0.83)$ & $0.79(0.63-0.98)$ \\
\hline High, $\geq 3$ & $0.74(0.68-0.81)$ & $0.90(0.79-1.04)$ & $0.69(0.61-0.77)$ & $0.93(0.79-1.09)$ \\
\hline \multicolumn{5}{|l|}{ Age } \\
\hline $15-39$ years & $0.98(0.60-1.60)$ & $0.82(0.42-1.60)$ & 0.00 & 0.00 \\
\hline 40-64 years & $0.78(0.72-0.84)$ & $1.07(0.95-1.21)$ & $0.77(0.61-0.97)$ & $0.89(0.65-1.21)$ \\
\hline $65-79$ years & $0.74(0.71-0.78)$ & $0.91(0.84-0.99)$ & $0.69(0.61-0.78)$ & $0.85(0.71-1.02)$ \\
\hline$\geq 80$ years & $0.80(0.75-0.85)$ & $0.85(0.76-0.95)$ & $0.75(0.66-0.85)$ & $0.95(0.77-1.16)$ \\
\hline Patients with indication for statin use $\mathrm{a}^{\mathrm{a}}$ & $0.70(0.67-0.73)$ & $0.78(0.72-0.85)$ & $0.70(0.64-0.77)$ & $0.86(0.75-1.00)$ \\
\hline \multicolumn{5}{|l|}{ Supplementary adjusted model for 2001-2009 } \\
\hline Receipt of current year's influenza vaccine before index date & $0.78(0.72-0.85)$ & $0.89(0.78-1.01)$ & $0.76(0.65-0.88)$ & $0.92(0.72-1.18)$ \\
\hline Preventive GP consultations and services & $0.77(0.69-0.86)$ & $1.10(0.94-1.29)$ & $0.80(0.66-0.97)$ & $0.92(0.70-1.19)$ \\
\hline Social medicine-related GP consultations & $0.73(0.66-0.81)$ & $0.91(0.77-1.08)$ & $0.82(0.72-0.94)$ & $0.96(0.79-1.18)$ \\
\hline GP conversational therapy & $0.80(0.61-1.04)$ & $0.87(0.59-1.29)$ & $0.89(0.67-1.19)$ & $0.66(0.41-1.05)$ \\
\hline Application for reimbursement for chronic illness & $0.43(0.23-0.81)$ & $0.43(0.16-1.22)$ & $0.64(0.47-0.86)$ & $0.86(0.54-1.35)$ \\
\hline
\end{tabular}

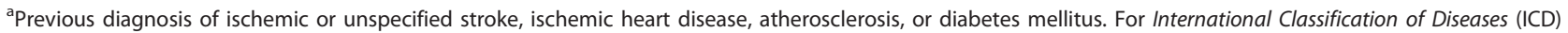
codes, see Additional file 4. Adjusted pneumonia hospitalization odds ratios (ORs) (case-control study) and 30-day mortality hazard ratios (HRs) (cohort study) associated with current and former use of statins are shown. $\mathrm{Cl}$, confidence interval; $\mathrm{GP}$, general practitioner. 

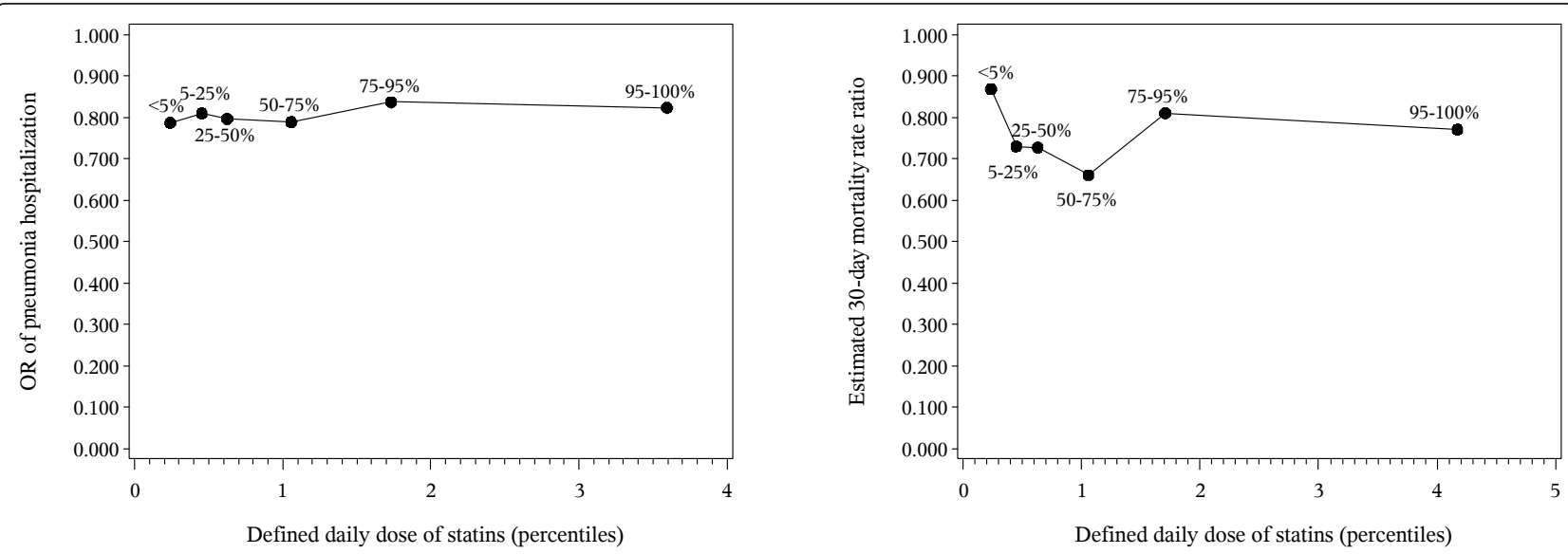

Figure 1 Statin dose and pneumonia risk and mortality. Dose-response associations between the number of defined daily doses (DDDs) of statins per day received within 125 days of the admission/index date and pneumonia hospitalization odds ratios (a) and hazard ratios for death (b). The relationship between DDDs and pneumonia was assessed by dividing DDDs into percentiles of users. OR, odds ratio.

use, but the risk of hospitalization for pneumonia was lower. This finding suggests that the improved prognosis in statin users was not due simply to surveillance bias resulting in a higher chance of being hospitalized for pneumonia, including milder pneumonia episodes. Our results are consistent with four published case-control studies of pneumonia risk in statin users compared with non-users [10-13]. All four studies used primary-care data and showed ORs ranging from 0.47 to 0.78 in favor of statin use.

In 2009, Dublin and colleagues [14] published a casecontrol study of 1,125 cases and 2,235 controls in a population of immunocompetent, community-dwelling group health members from 65 to 94 years old. The study showed that statin use was associated with an increased risk of pneumonia. Their baseline-adjusted model (age, sex, and calendar year) yielded an OR of 1.13 ( $95 \%$ CI 0.95 to 1.34 ), which was very close to our OR (1.17) matched for age, sex, urbanization of place of residence, and index date; the fully adjusted model (comorbid illnesses, lifestyle, demographic characteristics, and functional and cognitive status) yielded an OR of 1.26 (95\% CI 1.01 to 1.56). For cases leading to hospital admission (35\% of cases), the risk conferred by statin use was even greater (adjusted OR $=1.61,95 \%$ CI 1.08 to 2.39). Confounder data, obtained in part from medical record reviews, included information on functional and cognitive status. This information was used to explain findings and to suggest a strong unaccounted healthyuser effect in previous studies. However, their study was restricted to a selected group of older people. In our study, there was nothing to suggest that patients who used statins had a covariate profile associated with a good prognosis. The age- and sex-matched ORs for pneumonia risk and 30-day mortality associated with statin use were changed from harm to benefit after adjusting for the types of potential confounders included in the analysis carried out by Dublin and colleagues. This discrepancy may be due to differences in the available information on potential confounders or may indicate that the potential healthy-user effect associated with statin use is weaker in our Danish study population.

Although most studies have reported a substantial protective effect of statin use on pneumonia outcomes [16-22], the possibility of a healthy-user effect among statin users in hospitalized patients with pneumonia was proposed on the basis of a 2006 study by Majumdar and colleagues [23] that reported negative statin effects on outcomes. The limitations of this study, including the composite endpoint and the potential overadjustment for covariates (for example, pneumonia severity), have been discussed elsewhere $[5,26]$. A recent prospective cohort study of 1,895 hospitalized patients with pneumonia in the US also reported some evidence of a healthy-user bias but no decreased risk of severe sepsis (relative risk = $0.98,95 \%$ CI 0.71 to 1.36 ) or material reduction of 90 -day mortality in preadmission statin users (relative risk = $0.90,95 \%$ CI 0.63 to 1.29 ) [24]. However, the study suggested that continued inpatient statin therapy may reduce pneumonia mortality (adjusted OR $=0.73,95 \%$ CI 0.47 to 1.13) [24], a finding supported by results from Rothberg and colleagues [22] (adjusted OR $=0.90,95 \%$ CI 0.82 to 0.99 ) and Vandermeer and colleagues [21] (adjusted OR $=0.59,95 \%$ CI 0.38 to 0.92 ). Unfortunately, we did not have inpatient statin data. Hopefully, future trials will clarify whether continuation or initiation of statin therapy on hospital admission for pneumonia may improve outcome.

To date, there have been no randomized controlled trials large enough to determine the clinical efficacy of 
statins in patients with pneumonia. In a randomized controlled trial of 83 patients with suspected or documented bacterial infection, statin therapy was associated with a reduction in inflammatory cytokines, but the study was too small to address clinical endpoints [34]. Recently, post hoc analyses from the large ASCOT-LLA (Anglo-Scandinavian Cardiac Outcomes Trial-Lipid Lowering Arm) randomized controlled trial of patients with hypertension and additional cardiovascular risk factors suggested a protracted long-term legacy effect after atorvastatin treatment (median of 3.3 years), resulting in reduced long-term all-cause mortality 8 years after closure of the trial ( $\mathrm{HR}=0.85,95 \%$ CI 0.73 to 0.99 ). Interestingly, an important contributor to the decreased all-cause mortality was a reduction in deaths from infection and respiratory illness [35].

The main strengths of our study include its populationbased design and setting in a tax-supported universal health-care system free at the point of delivery, which reduce the potential for selection and referral bias. The positive predictive value of the DNRP with regard to pneumonia diagnosis is estimated to be $90 \%$ (95\% CI $82 \%$ to $95 \%$ ) [36], and the degree of completeness of the Danish prescription registries is excellent for reimbursed prescription drugs [37].

A possible study limitation is the use of filled prescriptions as a proxy for actual drug use. However, compliance with statin treatment in Denmark is good [38]. In addition, our observational study design may be vulnerable to uncontrolled confounding. As discussed above, a key question is whether statin users are healthier than non-users in terms of general health factors. Importantly, we found higher rates of most pneumonia risk and prognostic factors among statin users compared with nonusers in our analyses, including lifestyle-related diseases such as chronic pulmonary disease, cancer, and diabetes. We adjusted comprehensively for potential confounders, including a wide range of premedication drugs and comorbidities, recent surgery, living with small children, and socioeconomic indicators. To further assess possible residual confounding arising from a healthy-user effect, we retrieved information from the National Health Insurance Service Registry on influenza vaccination and markers for frailty and health awareness. This information, gleaned from GP consultations, is not available in most health registries. After these factors were taken into account, statin use was still associated with a clinically important reduction in pneumonia hospitalizations and deaths. Moreover, the prevalence of current statin use among Danish patients with pneumonia has more than doubled, from an average of $4.6 \%$ in $1997-2004$ to $10.2 \%$ in the updated study period (1997-2009), and statins have become an inexpensive treatment; both factors are likely to dilute any healthy-user effect. Finally, recent data from
Denmark showed no indication of a particularly healthy lifestyle associated with statin use. In a questionnairebased public health survey conducted in the central region of Denmark in 2006, people who used statins were more obese, exercised less, ate healthy foods more, and smoked and drank approximately the same amount as non-users [39].

\section{Conclusions}

Current statin use is associated with a clearly decreased risk of hospitalization for pneumonia and lower 30-day mortality following pneumonia. These results add to the evidence that treatment with statins may have a beneficial effect in reducing severity and possibly preventing pneumonia. Hospitalization rates for pneumonia and sepsis are currently rising in aging Western populations, and case fatality remains high. Our results should be confirmed in randomized statin therapy trials among patients with pneumonia and in statin prevention trials among individuals at risk for pneumonia since positive results would have important clinical and public health implications.

\section{Key messages}

- Statins exert beneficial pleiotropic effects that may decrease risk and improve outcomes of severe bacterial infections like sepsis and pneumonia.

- While awaiting large clinical trials that address this issue, we conducted the hitherto largest combined population-based case-control and cohort study, taking advantage of high-quality Danish health registries and using extensive adjustment for potential confounders.

- Current statin use was associated with a decreased risk of hospitalization for pneumonia and lower 30day mortality following pneumonia.

\section{Additional material}

Additional file 1: Appendix 1. International Classification of Diseases (ICD)-8 and 10 codes, used to identify pneumonia patients and comorbidities.

Additional file 2: Appendix 2. Anatomical Therapeutic Chemical (ATC) prescription codes, used to identify statins and other preadmission medications.

Additional file 3: Appendix 3. Service codes from the Danish National Health Insurance Service Registry, used to identify markers of frailty and health awareness.

Additional file 4: Appendix 4. Anatomical Therapeutic Chemical (ATC) prescription codes, used to identify patients with indication for statin use (Table 5).

\section{Abbreviations}

Cl: confidence interval; CRS: Civil Registration System; DDD: defined daily dose; DNRP: Danish National Registry of Patients; GP: general practitioner; HR: hazard ratio; ICD: International Classification of Diseases; OR: odds ratio. 


\section{Acknowledgements}

The study was supported by the Clinical Epidemiological Research Foundation, the Karen Elise Jensen Foundation, and the Danish Lung Association.

\section{Authors' contributions}

AGN helped to conceive the study idea, to design the study, to analyze the data, and to review the literature and wrote the first draft. HTS helped to conceive the study idea, to design the study, and to collect the data. SP helped to conceive the study idea and to design the study. RWT helped to conceive the study idea, to design the study, to analyze the data, and to review the literature. RBN and AHR helped to design the study, to collect the data, and to analyze the data. All authors interpreted the findings and read and approved the final manuscript.

\section{Competing interests}

The authors declare that they have no competing interests.

Received: 2 May 2012 Revised: 2 July 2012 Accepted: 12 July 2012 Published: 12 July 2012

\section{References}

1. Terblanche M, Almog Y, Rosenson RS, Smith TS, Hackam DG: Statins and sepsis: multiple modifications at multiple levels. Lancet Infect Dis 2007, 7:358-368

2. Niessner A, Steiner S, Speidl WS, Pleiner J, Seidinger D, Maurer G, Goronzy J, Weyand CM, Kopp CW, Huber K, Wolzt M, Wojta J: Simvastatin suppresses endotoxin-induced upregulation of toll-like receptors 4 and 2 in vivo. Atherosclerosis 2006, 189:408-413.

3. McGown CC, Brookes ZL: Beneficial effects of statins on the microcirculation during sepsis: the role of nitric oxide. Br J Anaesth 2007, 98:163-175.

4. Yunoki K, Nakamura K, Miyoshi T, Enko K, Kubo M, Murakami M, Hata Y, Kohno K, Morita H, Kusano KF, Ito H: Impact of hypertriglyceridemia on endothelial dysfunction during statin +/- ezetimibe therapy in patients with coronary heart disease. Am J Cardiol 2011, 108:333-339.

5. Chalmers JD, Short PM, Mandal P, Akram AR, Hill AT: Statins in community acquired pneumonia: Evidence from experimental and clinical studies. Respir Med 2010, 104:1081-1091.

6. Janda S, Young A, Fitzgerald JM, Etminan M, Swiston J: The effect of statins on mortality from severe infections and sepsis: a systematic review and meta-analysis. J Crit Care 2010, 25:656.e7-656.e22.

7. Kopterides P, Falagas ME: Statins for sepsis: a critical and updated review. Clin Microbiol Infect 2009, 15:325-334.

8. Falagas ME, Makris GC, Matthaiou DK, Rafailidis PI: Statins for infection and sepsis: a systematic review of the clinical evidence. J Antimicrob Chemother 2008, 61:774-785.

9. Tleyjeh IM, Kashour T, Hakim FA, Zimmerman VA, Erwin PJ, Sutton AJ, Ibrahim T: Statins for the prevention and treatment of infections: a systematic review and meta-analysis. Arch Intern Med 2009, 169:1658-1667

10. van de Garde EM, Hak E, Souverein PC, Hoes AW, van den Bosch JM, Leufkens HG: Statin treatment and reduced risk of pneumonia in patients with diabetes. Thorax 2006, 61:957-961.

11. Schlienger RG, Fedson DS, Jick SS, Jick H, Meier CR: Statins and the risk of pneumonia: a population-based, nested case-control study. Pharmacotherapy 2007, 27:325-332.

12. Myles PR, Hubbard RB, McKeever TM, Pogson Z, Smith CJ, Gibson JE: Risk of community-acquired pneumonia and the use of statins, ace inhibitors and gastric acid suppressants: a population-based case-control study. Pharmacoepidemiol Drug Saf 2009, 18:269-275.

13. Vinogradova Y, Coupland C, Hippisley-Cox J: Risk of pneumonia in patients taking statins: population-based nested case-control study. $\mathrm{Br} J$ Gen Pract 2011, 61:e742-748.

14. Dublin S, Jackson ML, Nelson JC, Weiss NS, Larson EB, Jackson LA: Statin use and risk of community acquired pneumonia in older people: population based case-control study. BMJ 2009, 338:b2137.

15. Thomsen RW, Riis A, Kornum JB, Christensen S, Johnsen SP, Sorensen HT: Preadmission use of statins and outcomes after hospitalization with pneumonia: population-based cohort study of 29,900 patients. Arch Intern Med 2008, 168:2081-2087.
16. Mortensen EM, Restrepo MI, Anzueto A, Pugh J: The effect of prior statin use on 30-day mortality for patients hospitalized with communityacquired pneumonia. Respir Res 2005, 6:82.

17. Frost FJ, Petersen H, Tollestrup K, Skipper B: Influenza and COPD mortality protection as pleiotropic, dose-dependent effects of statins. Chest 2007, 131:1006-1012.

18. Mortensen EM, Pugh MJ, Copeland LA, Restrepo MI, Cornell JE, Anzueto A, Pugh JA: Impact of statins and angiotensin-converting enzyme inhibitors on mortality of subjects hospitalised with pneumonia. Eur Respir J 2008, 31:611-617.

19. Chalmers JD, Singanayagam A, Murray MP, Hill AT: Prior statin use is associated with improved outcomes in community-acquired pneumonia. Am J Med 2008, 121:1002-1007, e1001.

20. Douglas I, Evans S, Smeeth L: Effect of statin treatment on short term mortality after pneumonia episode: cohort study. BMJ 2011, 342:d1642.

21. Vandermeer ML, Thomas AR, Kamimoto L, Reingold A, Gershman K, Meek J, Farley MM, Ryan P, Lynfield R, Baumbach J, Schaffner W, Bennett N, Zansky S: Association between use of statins and mortality among patients hospitalized with laboratory-confirmed influenza virus infections: a multistate study. J Infect Dis 2012, 205:13-19.

22. Rothberg MB, Bigelow C, Pekow PS, Lindenauer PK: Association between statins given in hospital and mortality in pneumonia patients. J Gen Intern Med 2012, 27:280-286.

23. Majumdar SR, McAlister FA, Eurich DT, Padwal RS, Marrie TJ: Statins and outcomes in patients admitted to hospital with community acquired pneumonia: population based prospective cohort study. BMJ 2006, 333:999.

24. Yende S, Milbrandt EB, Kellum JA, Kong L, Delude RL, Weissfeld LA, Angus DC: Understanding the potential role of statins in pneumonia and sepsis. Crit Care Med 2011, 39:1871-1878.

25. Dormuth CR, Patrick AR, Shrank WH, Wright JM, Glynn RJ, Sutherland J, Brookhart MA: Statin adherence and risk of accidents: a cautionary tale. Circulation 2009, 119:2051-2057.

26. Thomsen RW: The lesser known effects of statins: benefits on infectious outcomes may be explained by 'healthy user' effect. BMJ 2006, 333:980-981.

27. International Statistical Classification of Diseases, 10th Revision(ICD-10). Geneva, Switzerland:World Health Organisation;[http://apps.who.int/ classifications/icd10/browse/2010/en].

28. Dansk Lægemiddel Information A/S homepage. [http://medicin.dk], Danish.

29. Charlson ME, Pompei P, Ales KL, MacKenzie CR: A new method of classifying prognostic comorbidity in longitudinal studies: development and validation. J Chronic Dis 1987, 40:373-383.

30. de Groot V, Beckerman H, Lankhorst GJ, Bouter LM: How to measure comorbidity: a critical review of available methods. J Clin Epidemiol 2003, 56:221-229.

31. Deyo RA, Cherkin DC, Ciol MA: Adapting a clinical comorbidity index for use with ICD-9-CM administrative databases. J Clin Epidemiol 1992, 45:613-619.

32. Rothman KJ: Modern Epidemiology Philadelphia, PA: Lippincott Williams \& Wilkins; 1998.

33. Austin PC, Anderson GM, Cigsar C, Gruneir A: Comparing the cohort design and the nested case-control design in the presence of both time-invariant and time-dependent treatment and competing risks: bias and precision. Pharmacoepidemiol Drug Saf 2012, 21:714-724.

34. Novack V, Eisinger M, Frenkel A, Terblanche M, Adhikari NK, Douvdevani A, Amichay D, Almog Y: The effects of statin therapy on inflammatory cytokines in patients with bacterial infections: a randomized doubleblind placebo controlled clinical trial. Intensive Care Med 2009, 35:1255-1260.

35. Sever PS, Chang CL, Gupta AK, Whitehouse A, Poulter NR: The AngloScandinavian Cardiac Outcomes Trial: 11-year mortality follow-up of the lipid-lowering arm in the UK. Eur Heart J 2011, 32:2525-2532.

36. Thomsen RW, Riis A, Norgaard M, Jacobsen J, Christensen S, McDonald CJ, Sorensen HT: Rising incidence and persistently high mortality of hospitalized pneumonia: a 10-year population-based study in Denmark. J Intern Med 2006, 259:410-417.

37. Gaist D, Sorensen HT, Hallas J: The Danish prescription registries. Dan Med Bull 1997, 44:445-448. 
38. Larsen J, Andersen M, Kragstrup J, Gram LF: High persistence of statin use in a Danish population: compliance study 1993-1998. Br J Clin Pharmacol 2002, 53:375-378.

39. Thomsen RW, Nielsen RB, Nørgaard M, Horsdal HT, Larsen FB, Sørensen HT: Lifestyle profile among statin users. Presented at: 27th International Conference on Pharmacoepidemiology \& Therapeutic Risk Management; 14-17 August 2011; Chicago, IL. Abstract 258.

doi:10.1186/cc11418

Cite this article as: Nielsen et al:: The impact of statin use on pneumonia risk and outcome: a combined population-based casecontrol and cohort study. Critical Care 2012 16:R122.

Submit your next manuscript to BioMed Central and take full advantage of:

- Convenient online submission

- Thorough peer review

- No space constraints or color figure charges

- Immediate publication on acceptance

- Inclusion in PubMed, CAS, Scopus and Google Scholar

- Research which is freely available for redistribution

Submit your manuscript at www.biomedcentral.com/submit
C Biomed Central 